\title{
Power Quality aspects of Smart Grids
}

\author{
Math H.J. Bollen ${ }^{1,2}$, Jin Zhong ${ }^{3}$, Francisc Zavoda ${ }^{4}$, Jan Meyer ${ }^{5}$ Alex McEachern ${ }^{6}$, and Felipe Córcoles López ${ }^{7}$ \\ ${ }^{1}$ STRI AB, 77180 Ludvika, Sweden \\ ${ }^{2}$ Luleå University of Technology, 93187 Skellefteå, Sweden \\ ${ }^{3}$ The University of Hong Kong, Hong Kong \\ ${ }^{4}$ IREQ (Research Institute of Hydro-Québec), Québec, Canada \\ ${ }^{5}$ Technische Universität Dresden, Dresden, Germany \\ ${ }^{6}$ Power standards labs, San Francisco, USA \\ ${ }^{7}$ Politechnical University of Catalonia, Barcelona, Spain.
}

\begin{abstract}
This paper gives an overview of the smart grids developments, seen from a power-quality viewpoint; describes the main relations between smart grids and power quality, and goes into some detail for some of the aspects. Different types of real and virtual energy storage are distinguished. Power-quality monitoring in the smart grid is discussed in detail. Further aspects discussed in the paper are: emission by new devices; interference between devices and power-line communication; allocation of emission limits; improving voltage quality; immunity of devices; and weakening of the transmission grid. Although the smart grid brings many new power-quality challenges, these should not result in the introduction of unnecessary barriers against the introduction of new technology.
\end{abstract}

\section{Key words}

Smart grids; power quality, storage, power-quality monitoring, distribution automation, harmonics.

\section{Introduction}

There is at the moment no consistent definition of " $a$ smart grid" or "the smart grid". Different people use different definitions, and the definitions develop with time. In this paper, we will simply limit ourselves to a description, and not worry about a precise definition. The term "smart grid" refers to a way of operating the power system using communication technology, power electronic technologies, and storage technologies to balance production and consumption at all levels, i.e. from inside of the customer premises all the way up to the highest voltage levels [1][2]. An alternative way of defining the concept is as the set of technologies, whatever they may be, that are needed to allow new types of production and new types of consumption to be integrated in the electric power system

The concept of "smart grid" was started from a number of the technology innovations in the power industry. It is a result of the new technologies applied in power systems, including renewable energy sources generation, distributed generation, and the latest information and communication technology.

With the (technical and regulatory) developments of renewable energy generation technologies, the penetration level of especially wind power has becomes very high in some parts of the system. Similar developments are expected for solar power and domestic combined heat and power. However, the increase in intermittent, non-predictable and non-dispatchable energy generation puts highest requirements on power balance control, from primary control through operational planning. The traditional control and communication system needs to be improved to accommodate for a high penetration of renewable energy sources.

The term "microgrid" is used to describe a customerowned installation containing generation as well as consumption, where there is a large controllability of the exchange of power between the microgrid and the rest of the grid [3]. Such microgrids provide the possibilities of load-shifting and peak-shaving through demand side management. Consumers could use the electricity from their own sources or even sell electricity to the grid during the peaking periods, hence increase the energy efficiency and defer the investments in transmission and distribution networks. To perform demand response in a most efficient way, the market and system operation conditions need to be known. Smart meters / advanced metering infrastructure (AMI) and two-way communication technologies can provide consumers and operators the information for decision making.

The automation system of the traditional power system is still based on the design and operation of the system as it was decades ago. The latest developments in information and communication technologies have only found very limited implementation in the power system automation. One of the objectives of smart grid is to update the power 
system automation (including transmission, distribution, substation, individual feeders and even individual customers) using the latest technology.

Besides technology innovations, another important reason for smart grid is to improve the services in power supply to consumers. Through AMI (also known as "smart meters"), consumers are no longer passive consumers. They can monitor their own voltage and power and manage their energy consumption for example based on the electricity prices. Feedback on consumption is also seen as an important tool for energy saving.

\section{Balancing Production and Consumption}

Any amount of production or consumption can be connected at any location in the power system provided the difference between these two remains within a certain band. The unbalance between production and consumption at a certain location is provided by the transfer capacity from the rest of the system. The situation can be more complicated in meshed systems, but this is the basic rule.

Traditionally, production capacity and consumption demand have been seen as independent of each other. So the traditional grid has been designed to cope with the maximum amount of production, and also with the maximum amount of consumption. This approach sets hard limits on both production and consumption.

A "smart grid" that can control, or influence, both production and consumption would allow more of both to be integrated into the power system.

To accomplish this goal, communication technology may be order to inform or encourage changes in production (i.e. generator units) and consumption (i.e. customers or devices). Most published studies propose some kind of market mechanism to maintain balance between production and consumption, but more direct methods are also possible, with either the network operator or an independent entity taking control.

Different methods are available to balance consumption and production while at the same time optimizing energy efficiency, reliability and/or power quality.

$\checkmark \quad$ Physical energy storage, for example in the form of batteries or pumped-storage hydro. Such storage could be owned and operator by a customer (an enduser or a generator company), owned by a customer and operated by the network operator, or owned and operated by the network operator.

$\checkmark$ Virtual energy storage, by shifting of energy consumption to a later or earlier moment in time. Charging of car batteries is often mentioned, but this method of virtual storage can also be used for cooling or heating loads. It is important to realize that this approach does not result in energy saving, but in more efficient use of the generation facilities and the power system transport capacity. The total energy consumption may be reduced somewhat, for example by reduced losses, reduced average temperatures with heating systems (increased with cooling ), and the ability to use more efficient forms of energy, but these are minor effects and they should not be seen as the main reason for introducing the new technology. $\checkmark \quad$ Load shedding, where load is removed from the system when all other methods fail. This method is available now but is rarely used in most countries. Accepting the occasional small amount of load shedding may, in some cases save large investments in the power system. (In some developing countries, uncontrolled and inadvertent load shedding often occurs automatically during grid or generator overload, but this is a poor example of load shedding, and hopefully only a temporary situation.) Under-frequency load shedding, as used in almost all systems, can be seen as an extreme case of reserve capacity in the form of load shedding. This is not the kind of application that is normally considered in the discussion on smart grids.

$\checkmark$ Curtailment of production: For renewable sources like sun and wind, the primary energy is usually transformed into electricity whenever it is available. But if generation exceeds consumption, renewable sources may be turned off, or curtailed. The term "spilled wind" is sometimes used to express this concept.

$\checkmark \quad$ Shifting of production: for sources like natural gas (for combined-heat-and-power) or hydro power, the primary energy source can be temporarily stored, then used at a later time. Not using the primary energy sources will make it available at a later time.

\section{Power quality}

In the ongoing discussions about smart grids, power quality has to become an important aspect and should not be neglected. An adequate power quality guarantees the necessary compatibility between all equipment connected to the grid. It is therefore an important issue for the successful and efficient operation of existing as well as future grids. However power quality issues should not form an unnecessary barrier against the development of smart grids or the introduction of renewable sources of energy. The "smart" properties of future grids should rather be a challenge for new approaches in an efficient management of power quality. Especially the advanced communication technologies can establish new ways for selective power quality management.

Power quality covers two groups of disturbances: variations and events [4]. While variations are continuously measured and evaluated, events occur in general unpredictable and require a trigger action to be measured. Important variations (cf. EN 50160) are: slow voltage changes, harmonics, flicker and unbalance. Important events are rapid voltage changes, dips, swells and interruptions.

The actual power quality (i.e. the disturbance levels) results from the interaction between the network and the connected equipment (cf. Fig. 1). 


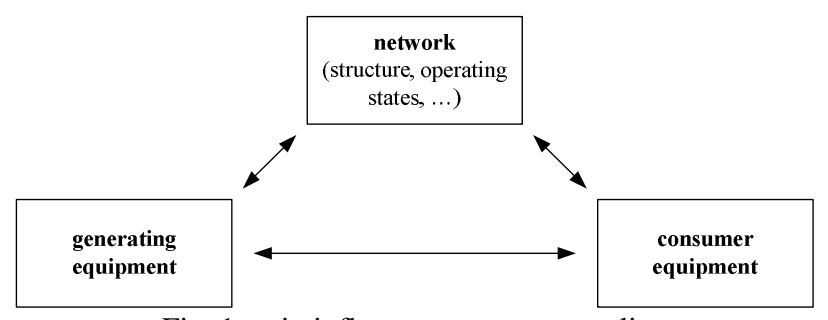

Fig. 1 main influences on power quality.

All three areas are expected to see significant changes in the future. This means that power quality issues will also change with the consecutive development of future grids. The following comments shall give some examples for possible future developments in power quality. Further expected developments are also discussed in Section 5.

\section{a) Generating equipment}

The penetration of microgeneration (typically defined as generation with a rated power of less than 16 A per phase) in the low-voltage networks is expected to increase continuously. In domestic installations this will be mainly single phase equipment based on selfcommutating inverters with switching frequencies in the range of several $\mathrm{kHz}$. Emissions in the range of low order harmonics can usually be neglected. The emissions shift into the range of higher frequencies, possibly between 2 and $9 \mathrm{kHz}$, where a serious discussion is needed on the choice of appropriate limits. We will come back to this in Section 5.1.

Furthermore micro-generation equipment will often be connected single-phase. This could increase the negativesequence and zero-sequence voltage in the low-voltage grid. In weak distribution networks, existing limits could be exceeded rather quickly. Reconsidering the limits for negative-sequence voltages and introducing limits for zero-sequence voltage could be possible needed.

\section{b) Consumer equipment}

The introduction of new and more efficient technologies is the main driver for changes in consumer equipment.

One widely-discussed example is the change from incandescent lamps to energy saving lamps. Compact fluorescent lamps are at the moment the main replacement for incandescent lamps, but they are probably only an intermediate step before the LEDtechnique will become widely accepted. Seen from the network, each of the new lamp technologies results in the replacement of a resistive load by a rectifier load. The fundamental current is reduced significantly whereas the harmonic currents are increased. High penetration together with high coincidence of operation may lead to an increase of low order harmonics. Several network operators fear an increase of especially the fifth harmonic voltage above the compatibility levels. Discussion is ongoing in IEC working groups about the need for additional emission requirements on new types of lighting of low wattage. The same would hold for other improved (energy-efficient drives) or new (photovoltaics, battery chargers for electric and hybrid cars) equipment.

As mentioned before, such limits should however not result in unnecessary barriers against the introduction of new equipment. Alternative paths, like an increase of the compatibility levels for some higher harmonics, should at least be considered.

\section{c) Distribution network}

The short-circuit power is an important factor in power quality management. Under constant emission a higher short circuit power results in a better voltage quality. Today the short-circuit power is mainly determined by the upstream network. In the IEC electromagneticcompatibility standards standards a reference impedance (cf. IEC 60725) is used as a link between compatibility levels (voltages) and emission limits (currents). In future grids with high penetration of generation significant differing supply scenarios may be possible, from supply by a strong upstream network to an islanded (selfbalanced) operation. This may lead to a significantly higher variability in short circuit power than today. Thus the approach based on fixed reference impedances may be inadequate or the use of high emitting loads may only be acceptable for certain operational states of the network or only in conjunction with power quality conditioners (owned by a customer, by the network operator, or by a third party).

Due to the continuous decrease of resistive loads providing damping stability issues may become important for low-voltage networks too. In conjunction with increasing capacitive load (the EMC filters of electronic equipment) resonance points with decreasing resonant frequencies as well as lower damping can appear.

\section{Power-quality monitoring}

Growing service quality expectations and reduced possibilities for grid enforcements make advanced distribution automation (ADA) an increasingly necessary development for network operators and the next large step in the evolution of the power systems to smart grids. The management of the distribution system is mainly based on the information collected from the power flows by an integrated monitoring system. This enables realtime monitoring of grid conditions for the power system operators. It also enables automatic reconfiguration of the network to optimize the power delivery efficiency and to reduce the extent and duration of interruptions. The basic part of the monitoring system infrastructure is based on sensors, transducers, intelligent electronic devices (IED) and (revenue) meters collecting information throughout the distribution system.

A number of network operators have already proposed that the smart grid of the future should include:

$\checkmark$ Network monitoring to improve reliability,

$\checkmark$ Equipment monitoring to improve maintenance,

$\checkmark$ Product (power) monitoring to improve PQ.

In order to achieve these goals, the actual distribution system infrastructure (especially meters and remotely controlled IEDs) should be used to gather as much information as possible related to network, equipment and product (i.e. power quality and reliability) to improve the distribution system overall performance.

Among the most important ADA operating systems, that a smart grid will include, it can be mentioned:

$\checkmark$ Volt \& var control (VVC), 
$\checkmark \quad$ Fault location (FL),

$\checkmark$ Network reconfiguration or self-healing.

Network operators with an ambitious energy efficiency program have focused on two targets:

$\checkmark$ Capacitor banks installation,

$\checkmark$ Voltage control.

There is also another important goal: to reduce the duration of interruptions. To answer to these challenges, pilot projects are being conducted on conservation voltage reduction and fault location based on power quality related measurements provided by IEDs and revenue meters.

The VVC system requires a permanent monitoring of the voltage magnitude (averaged over 1 to $5 \mathrm{~min}$ ) at the end of the distribution feeder and the installation of switched capacitor banks. Besides that, the monitoring allows the detection of power quality disturbances such as longduration undervoltages and overvoltages, and voltage and current unbalance.

Basically, the voltage regulation system at the substation is replaced with an intelligent system that uses network measurements to maintain a voltage magnitude for all customers within the acceptable upper and lower limits. The VVC system also analyzes the reactive-power requirements of the network and orders the switching of capacitor banks when required.

An important goal is to prevent potential power quality problems due to the switching operations of capacitor banks (with rating up to 1.2 Mvar). [13].

Another goal was to evaluate the joint impact of the VVC system and voltage dips occurring on the grid. The results of the study indicate that the impact can be quantified by two effects:

$\checkmark$ Increasing number of shallow voltage dips is expected. Voltage reduction from 2 to $4 \%$ is obtained due to VVC system. Added to this is the voltage drop due to the fault: drops of 6 to $10 \%$ (not counted as dips) become drops of 10 to $12 \%$ (which are counted as dips).

$\checkmark$ Equipment malfunctioning or tripping: the joint contribution of the VVC system and the disturbance brings the residual voltage level below a critical threshold, around $70 \%$ of the nominal voltage for many devices.

Fault location is based either on a voltage drop fault location technique that uses waveforms from distributed power quality measurements along the feeder or on a fault current technique based on the measurement of the fault current at the substation. According to [14] the average error in locating the fault with the first technique was less than $2 \%$, in terms of the average main feeder length. An accurate fault-location technique results in a significant reduction in the duration of (especially) the longer interruptions. The information collected by the fault-location system can also be used for calculating dip related statistics and help to better understand the grid behaviour [15].

The third application, network reconfiguration or self healing, is based either on local intelligence (belonging to major distribution equipment controllers) or on decisions taken at the power system control centre, which remotely controls and operates the equipment used for network reconfiguration (reclosers and switches).
The impact of these applications on the distribution network and its customers is permanently evaluated.

The infrastructure belonging to ADA systems can be shared by a power-quality monitoring system capable of real time monitoring.

Depending on the type of ADA application or system, the monitoring can be done either at low-voltage or at medium-voltage level. In the first case monitoring devices may belong to an Advanced Metering Infrastructure (AMI) and in the second case they may belong to the distribution major equipment itself (i.e. controllers) (see Fig. 2) [16].

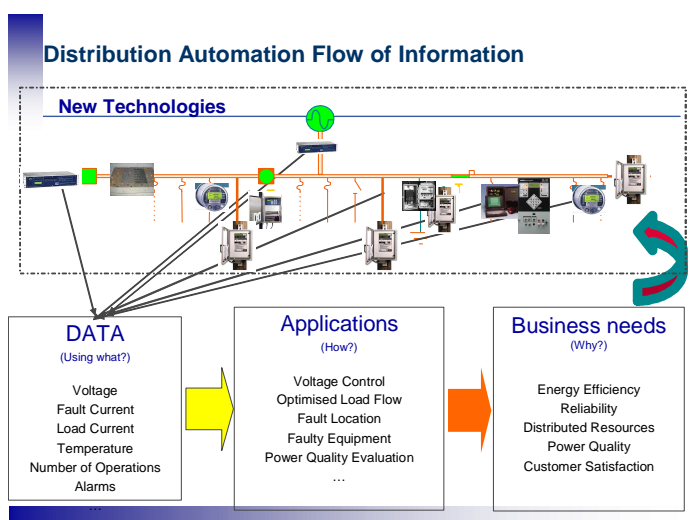

Fig. 2. Distribution Automation flow of information

The smart grid will allow a continuous power-quality monitoring that will not improve directly the voltage quality but will detect quality problems helping to mitigate them.

\section{Different Power-Quality Issues}

\subsection{Emission by new devices}

When smart grids are introduced, we expect growth both in production at lower voltage levels (distributed generation) and in new types of consumption (for example, charging stations for electric vehicles, expanded high-speed railways, etc.). . Some of these new types of consumption will emit power-quality disturbances, for example harmonic emission. Preliminary studies have shown that harmonic emission due to distributed generation is rather limited. Most existing end-user equipment (computer, television, lamps, etc) emit almost exclusively at the lower odd integer harmonics $(3,5,7,9$ etc), but there are indications that modern devices including certain types of distributed generators emit a broadband spectrum [6][7][8][9][12]. Using the standard methods of grouping into harmonic and interharmonic groups and subgroups below $2 \mathrm{kHz}$ will result in high levels for even harmonics and interharmonics. For frequencies above $2 \mathrm{kHz}$ high levels have been observed for the 200-Hz groups. An example is shown in Fig. 3: the spectrum of the emission by a group of three fullpower converter wind turbines, where $1 \mathrm{~A}$ is about $1 \%$ of 
the rated current.

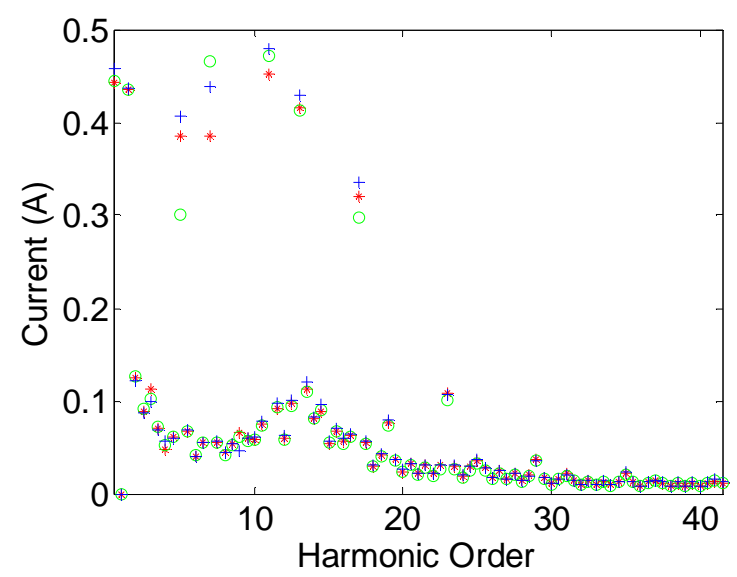

Fig. 3. Spectrum of the current for a group of three wind turbines: $95 \%$ values of the harmonic and interharmonic subgroups. The three colours and symbols refer to the three phases.

The emission is low over the whole spectrum, being at most $0.5 \%$ of the nominal current. The combination of a number of discrete components at the characteristic harmonics (5 and 7, 11 and 13, 17 and 19, etc) together with a broadband spectrum over a wide frequency range, is also being emitted by other equipment like energyefficient drives, microgenerators, and photo-voltaic installations. The levels are not always as low as for the example shown here. The existing compatibility levels are very low for some frequencies, as low as $0.2 \%$. Harmonic resonances are more common at these higher frequencies so that any reference impedance for linking emission limits to compatibility levels should be set rather high. Keeping strict to existing compatibility limits and existing methods of setting emission limits could put excessive demands on new equipment.

The measurement of these low levels of harmonics at higher frequencies will be more difficult than for the existing situation with higher levels and lower frequencies. This might require the development of new measurement techniques including a closer look at the frequency response of existing instrument transformers.

The presence of emission at higher frequencies than before also calls for better insight in the source impedance at these frequencies: at the point of connection with the grid as well as at the terminals of the emitting equipment.

\subsection{Interference between devices and power- line-communication}

Smart grids will depend to a large extent on the ability to communicate between devices, customers, distributed generators, and the grid operator. Many types of communication channels are possible. Power-line communication might seem an obvious choice due to its easy availability, but choosing power-line communication could introduce new disturbances in the power system, resulting in a further reduction in power quality. Depending on the frequency chosen for powerline communication, it may also result in radiated disturbances, possibly interfering with radio broadcasting and communication.
It is also true that modern devices can interfere with power-line-communication, either by creating a high disturbance level at the frequency chosen for power-line communication, or by creating a low-impedance path, effectively shorting out the power-line communication signal. The latter seems to be the primary challenge to power-line communication today [11].

So far, there have been no reports of widespread interference with sensitive equipment caused by powerline-communication, but its increased use calls for a detailed study.

\subsection{Allocation of Emission Limits}

When connecting a new customer to the power system, an assessment is typically made of the amount of emission that would be acceptable from this customer without resulting in unacceptable levels of voltage disturbance for other customers. For each new customer a so-called emission limit is allocated. The total amount of acceptable voltage distortion is divided over all existing and future customers. This assumes however that it is known how many customers will be connected in the future [17].

With smart grids, the amount of consumption will have no limit provided it is matched by a similar growth in production. This continued growth in both production and consumption could lead to the harmonic voltage distortion becoming unacceptably high. Also the number of switching actions will keep on increasing and might reach unacceptable values. One may say that production and consumption are in balance at the power-system frequency, but not at harmonic frequencies.

Another way of looking at this is that the system strength is no longer determined by the maximum amount of consumption and/or production connected downstream, but by the total amount of harmonic emission coming from downstream equipment. This will require a different way of planning the distribution network.

\subsection{Improving Voltage Quality}

One aim of smart grids is to improve the performance of the power system (or to prevent deterioration) without the need for large investments in lines, cables, transformers, etc.

From a customer viewpoint, the improvements can be in terms of reliability, voltage quality or price. All other improvements (e.g. in loading of cables or transformers, protection coordination, operational security, efficiency) are secondary to the customer.

Improvements in reliability and price are discussed in detail in several other papers and beyond the scope of this paper. The only voltage-quality improvement expected to be made by smart grids in the near future would be a reduction in longer-term voltage-magnitude variations. In theory, both undervoltages and overvoltages might be mitigated by keeping the correct local balance between production and consumption [18][19]. For rural networks, overvoltages and undervoltages are the main limitation for increasing consumption and production. These networks should therefore be addressed first.

The same balance between "production" and "consumption" can in theory also be used for the control 
of harmonic voltages. When the harmonic voltage becomes too large, either an emitting source could be turned off, or a harmonic filter could be turned on, or a device could be turned on that emits in opposite phase (the difference between these solutions is actually not always easy to see). Smart grid communication and control techniques, similar to those used to balance consumption and production (including market rules), could be set up to reduce harmonic emissions. This could be a solution for the growing harmonic emission with growing amounts of production and consumption.

Microgrids with islanding capability can, in theory, mitigate voltage dips by going very quickly from gridconnected operation to island operation.

The presence of generator units close to the loads allows the use of these units in maintaining the voltage during a fault in the grid.

\subsection{Immunity of devices}

Simultaneous tripping of many distributed generators due to a voltage-quality disturbance (like a voltage dip) is the subject of active discussion [5][10]. This problem is far from solved. As a smart grid attempts to maintain a balance between production and consumption, mass tripping of consumption could have similar adverse consequences. This should be further investigated.

\subsection{Weakening of the transmission grid}

The increased use of distributed generation and of large windparks will result in a reduction of the amount of conventional generation connected to the transmission system. The fault level will consequently be reduced, and power-quality disturbances will spread further. This will worsen voltage dips, fast voltage fluctuations (flicker) and harmonics. The severity of this has been studied for voltage dips. The conclusion from the study is that even with $20 \%$ wind power there is no significant increase in the number of voltage dips due to faults in the transmission system [20].

\section{Conclusions}

The new technology associated with smart grids offers the opportunity to improve the quality and reliability as experience by the customers. It will however also result in the increase of disturbance levels in several cases and thereby introduce a number of new challenges. But these new challenges should definitely not be used as arguments against the development of smart grids. However they should attract attention to the importance of power quality for the successful and reliable operation of smart grids. New developments need new approaches and perspectives from all parties involved (network operators, equipment manufacturers, customers, regulators, standardization bodies, and others).

\section{References}

[1] The smart grid - an introduction, (US) Department of Energy, 2008. http://www.oe.energy.gov/SmartGridIntroduction.htm.
[2] SmartGrids, European technology platform for the electricity networks of the future. http://www.smartgrids.eu/

[3] S.P.Chowdhury, P.Crossley, S.Chowdhury, Microgrids and active distribution networks. London: The Institution of Energy and Technology, 2009.

[4] M. Bollen, I. Gu, Signal processing of power-quality disturbances. New York: IEEE Press, 2006.

[5] J. Deuse, S. Grenard, M.H.J. Bollen, EU-DEEP integrated proect - Technical implications of the "hosting-capacity" of the sytem for DER, Int Journal of Distributed Energy Resources, Vol.4, No.1 (2008), pp.17-34.

[6] M.H.J. Bollen, P.F. Ribeiro, E.O.A. Larsson, C.M. Lundmark, Limits for voltage distortion in the frequency range 2-9 kHz, IEEE Transactions on Power Delivery, Vol.23, No.3 (July 2008), pp.1481-1487.

[7] S. T. Tentzerakis, and S. A. Papathanassiou, 'An Investigation of the Harmonic Emissions of Wind Turbines,' IEEE Trans. Energy Convers., vol. 22, no. 1, pp. 150-158, Mar. 2007.

[8] S. A. Papathanassiou, and M. P. Papadopoulos, 'Harmonic analysis in a power system with wind generation,' IEEE Trans. Power Del., vol. 21, no. 4, pp. 2006-2016, Oct. 2006.

[9] J. Plotkin, R. Hanitsch, and U. Schaefer, 'Power conditioning of a 132 MW wind farm,' in European Conference on Power Electronics and Applications, Sep. 2007, pp. 1-9.

[10] M.H.J. Bollen, M. Häger, Power quality: interaction between distributed energy resources, the grid and other customers, Electric Power Quality and Utilisation, Magazine Vol.1, No.1, 2005.

[11] S. K. Rönnberg, M. Wahlberg, E.O.A. Larsson, M.H. J. Bollen, C.M. Lundmark, Interaction between equipment and Power Line Communication: 9-95 kHz, Bucharest Power Tech, July 2009.

[12] M.H.J. Bollen, L.-Z. Yao, S.K. Rönnberg, M. Wahlberg, Harmonic and Interharmonic Distortion due to a windpark, IEEE PES General Meeting 2010.

[13] F. Zavoda, C. Perreault, A. Lemire, The impact of a volt \& var control system (VVC) on PQ and customer's equipment, IEEE PES T\&D Conference 2010

[14] M. Tremblay, R. Pater, F. Zavoda, G. Simard, Accurate fault-location technique based on distributed power-quality measurements, CIRED Conference 2007.

[15] F. Zavoda, M.H.J. Bollen, M. Tremblay, The behaviour of power distribution feeder dips, CIRED Conference 2009.

[16] F. Zavoda, G. Simard, Performance of today's intelligent controllers and meters, elements of an integrated monitoring system for ADA, IEEE PES General Meeting 2008.

[17] M. Bollen, M. Häger and M. Olofsson, Allocation of emission limits for individual emitters at different voltage levels: flicker and harmonics, CIGRE Sessions, Paris, August 2010, paper C4.106.

[18] T. Senjyu, Y. Miyazato, A. Yona, N. Urasaki, and T. Funabashi. Optimal distribution voltage control and coordination with distributed generation. IEEE Transactions on Power Delivery, 23(2):1236-1242, April 2008.

[19] I. Wasiak, M.C. Thoma, C.E.T. Foote, R. Mienski, R. Pawelek, P. Gburczyk, and G.M. Burt. A power-quality management algorithm for low-voltage grids with distributed resources. IEEE Transactions on Power Delivery, 23(2):1055-1062, April 2008.

[20] M.H.J. Bollen, M. Speychal, K. Lindén, Impact of increasing amounts of wind power on the dip frequency in transmission systems, Nordic Wind Power Conference, Helsinki, Finland, May 2006. 